Research Article

\title{
Loading History Effect on Stress Relaxation of Rock Joints
}

\author{
Guanghui Tian $\mathbb{D}^{1},{ }^{1}$ Weijian Liu $\mathbb{D}^{1},{ }^{1}$ Haohao $M a,{ }^{1}$ and Qingzhao Zhang ${ }^{2}$ \\ ${ }^{1}$ The School of of Architecture and Engineering, Zhongyuan University of Technology, Zhengzhou 450007, China \\ ${ }^{2}$ Department of Geotechnical Engineering, Tongii University, Shanghai, 200092, China
}

Correspondence should be addressed to Guanghui Tian; ghtian@zut.edu.cn

Received 30 April 2021; Accepted 26 October 2021; Published 15 November 2021

Academic Editor: Fan Gu

Copyright $\odot 2021$ Guanghui Tian et al. This is an open access article distributed under the Creative Commons Attribution License, which permits unrestricted use, distribution, and reproduction in any medium, provided the original work is properly cited.

In this study, a data analysis method for the relaxation test was proposed under step loading by conducting creep and stress relaxation tests of dentate discontinuity poured by cement mortar using biaxial creep machine under step loading and single-stage loading. First, a superposition method for the stress relaxation curve was deduced based on the data processing method of creep test by considering the effect of loading history on the relaxation curve and the characteristic of relaxation was analyzed. Second, this superposition method was verified by testing data under step loading and single-stage loading. Finally, this paper indicate the accurate way for the processing of relaxation test data under step loading. The results show that under the same stress level, the values of relaxation curve after the superposition are different from that under the single-stage loading test. Furthermore, the relaxation curves in the step loading test under different stress levels are similar to those in single-stage loading under the same stress level. Furthermore, creep deformation increase monotonically during the creep, but stress do not exhibit such a trend during relaxation, which is inconsistent with the Boltzmann superposition principle. The relaxation curve in the step loading test under different shear stress could reflect the relaxation properties of the single-stage loading test under the same shear stress, and unlike the data processing method of step loading creep test, superimposition is not needed.

\section{Introduction}

Rock mass is a complex geological body, and the existence of structural planes such as fractures and joints is the source of discontinuities, anisotropy, and heterogeneity in rock engineering [1]. Consequently, mechanical properties of rocks not only include elasticity and plasticity but also time-dependent behavior. Several engineering studies have shown that the failure and instability of rock mass does not occur immediately after the excavation is completed. The deformation characteristic of rock mass is affected by the stressstrain state, and the deformation occurs and adjusts with time continuously for a long period of time [2]. The timevarying nature of the stress-strain state of a rock mass is defined as the rheology of the rock mass. The indoor rheological test is a vital method for studying the rheological mechanical properties of rocks, which includes two commonly used test methods. The first method is the single-stage loading method, where the sample is directly loaded to a certain stress level during the test, which avoids the impact of loading history. However, it is rarely used because it is time consuming and requires many samples. The second method is the step loading method in which the load is divided into several levels during the test, and the rheological test is performed until the predetermined load is loaded. Although this method cannot avoid the impact of loading history, it is convenient and timesaving; besides, it avoids the effect of sample dispersion on the results of the rheological test.

The rheological properties of rocks comprise two aspects: creep and stress relaxation. Since the step loading method is convenient and timesaving, it is widely used in creep tests. For example, Liu et al. [3], Song et al. [4], Xiao et al. [5], Xie et al. [6], and Zhang et al. [7] used this method to perform the creep test on different types of rocks. The existence of structural planes significantly affects the mechanical properties of rock masses. Many researchers such as Xu et al. [8], Shen et al. [9], Zhang et al. [10], Wang et al. [11], and Lin et al. [12] used the step loading method to study the creep characteristics of structural planes. By analyzing the effect of 
loading history on the deformation of rock specimens based on the data obtained from shale multi-stage cyclic singlepump compression creep tests, Xia et al. [13] proposed a method for collating the creep test data. The "Chen's loading method" proposed by Chen and Kang can be used to infer the creep curve of the load under one-step loading through a mapping method [14, 15]. This method can establish the superposition relationship of the true deformation process, and it has been widely accepted and used in geotechnical rheology research globally.

Stress relaxation is another important aspect of rheology. Roadways and underground engineering are often damaged by relaxation of surrounding rocks, which is commonly observed [16]. Stress relaxation uses a constant deformation while the stress on the specimen is monitored as a function of time, which is difficult to achieve in existing experimental machines, so there are fewer experimental studies on stress relaxation [17]. In addition, the current studies on stress relaxation of rocks are mostly based on the creep test method. For example, Yu et al. [18], Chen et al. [19], and Paraskevopoulou et al. [20] performed uniaxial and triaxial relaxation tests on cylindrical rock specimens using the step loading method. Moreover, the step loading method is also used to study the relaxation characteristics of structural surfaces [21-24]. With the continuous improvement of test equipment and technology along with the growing importance of stress relaxation in engineering, several researchers are focusing on the stress relaxation characteristics of rocks. They have implemented a test method that is similar to the creep test, which is based on the step loading method. However, the studies on the data processing methods for the relaxation test under step loading are relatively scarce, e.g., like the data processing method of creep test, whether there is a method to deduce the relaxation curve of one-time loading through step loading have not been explored yet.

In engineering applications, the mechanical properties of structural planes usually control the stability of the rock engineering. Numerous studies have been carried out to investigate the mechanical behaviors of structural planes of rock mass [25-30]. In consideration on the complexity of structural plane, literature usually simplifies the rough structural plane into regularly dentate discontinuity with different values of the slope angle. Patton [31] was the first researcher in rock mechanics to relate the shear behavior of joints to normal load and roughness. His work is based on an idealized model of a joint in which roughness is represented by a series of constant-angle triangles or saw-teeth. Barton [32] has studied the behavior of natural rock joints and proposed a criterion that is modified from Patton. Other scholars also studied the mechanical properties of regular toothed discontinuity with different values of the slope angle in the past decades [24, 33-37]. Meanwhile, regular toothed discontinuity can be found in practical engineering, such as the headscarp of the Aishihik River Landslide [38].

To address this issue, cement mortar is used to artificially cast the samples with regular toothed structural surfaces at different angles to reduce the dispersion of test results. In this study, first, the creep test and stress relaxation test under step loading are performed on samples with regular toothed structural surface. Simultaneous, we have conducted the single-stage loading creep test and relaxation test under corresponding shear stress levels. Furthermore, a data processing method for the relaxation test under step loading is deduced from a theoretical perspective considering the influence of loading history, and the results are verified based on the test data. Then, the similarities and differences between the creep curve and relaxation curve according to the superposition principle are analyzed, and an appropriate method for the relaxation test under step loading is proposed. This knowledge resolves the outstanding issues of creep and relaxation equivalence, thereby enriching the theoretical understanding of rock mechanics.

\section{Materials and Methods}

2.1. Equipment. The test is performed on the CSS-1950 rock biaxial rheological testing machine manufactured by Changchun Testing Machine Research Institute at Tongji University (Figure 1). The maximum vertical and horizontal pressure applied by the testing machine are 500 and $300 \mathrm{kN}$, respectively, and these pressures can be applied simultaneously or separately. The vertical and horizontal biaxial deformation values of the sample can be measured simultaneously, where the measurement range is $\pm 3 \mathrm{~mm}$ and the measurement accuracy is $0.001 \mathrm{~mm}$. The temperature and humidity of the room remained essentially constant during the test.

2.2. Sample Preparation. All samples were formed with the same material, mixing ratio, and curing time to avoid large dispersion of test results due to uneven sample materials. The dimensions of the complete cube sample and the regular sample with the saw-tooth structure were $10 \mathrm{~cm} \times 10 \mathrm{~cm} \times 10 \mathrm{~cm}$, where the length of single tooth in the latter sample was $10 \mathrm{~mm}$ and the number of tooth shapes was 10 with three climbing angles: $10^{\circ}, 30^{\circ}$, and $45^{\circ}$. The schematics of the sample at $10^{\circ}$ and $30^{\circ}$ climbing angle are shown in Figure 2. The model materials were slag silicate $32.5 \mathrm{R}$ cement, standard sand, and water, and they were mixed in water:cement:sand ratio of $1: 2: 4$. This mixture was then stirred. Subsequently, it was placed into a steel mold and tapped to smoothen the surface for reducing the adverse effects during the sample preparation. Finally, the sample was disassembled after $24 \mathrm{~h}$ and cured for 28 days.

2.3. Testing and Loading Methods. Three complete cube samples were taken for the uniaxial compression test to obtain an average strength of $19.62 \mathrm{MPa}$. Subsequently, 10\% and $20 \%$ of the average uniaxial compressive strength of the complete cube sample, i.e., 1.962 $\mathrm{MPa}$ and 3.924 MPa, were selected as the normal stress during the creep and relaxation tests under the step loading and single-stage loading.

Before the creep and relaxation tests, three specimens with climbing angles of $10^{\circ}, 30^{\circ}$, and $45^{\circ}$ were selected for direct shear tests, and the peak values of the shear stress and shear displacement curves were considered as the shear strength. The average shear strength of the structural surface 


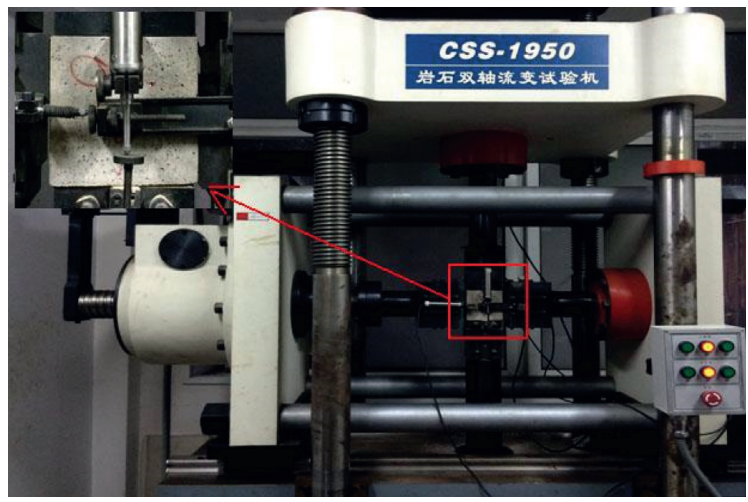

FIgURE 1: Snapshot of the test equipment.

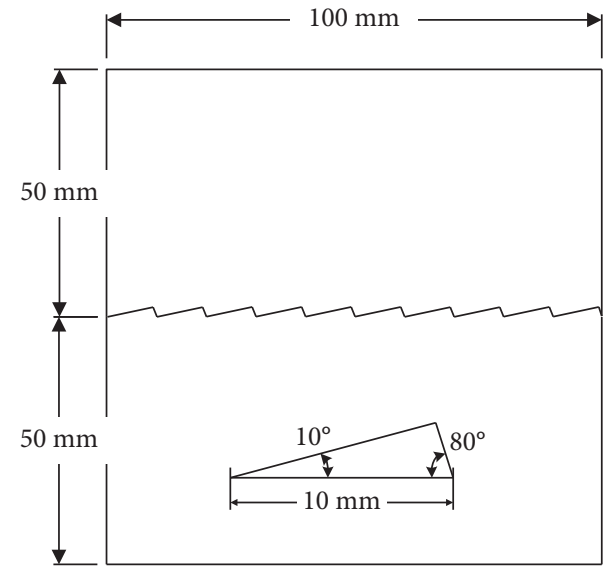

(a)

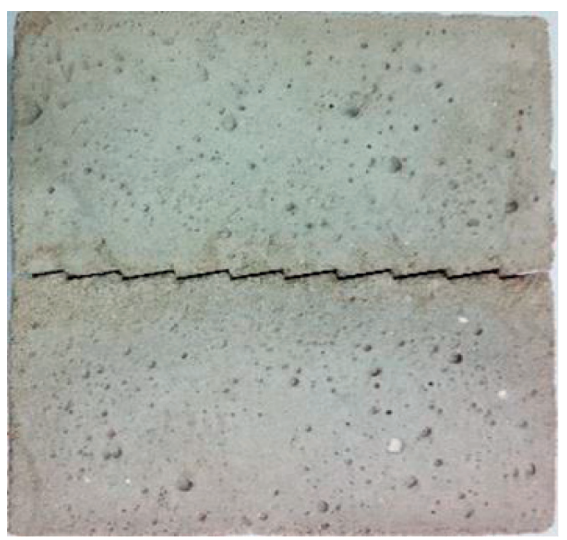

(c)

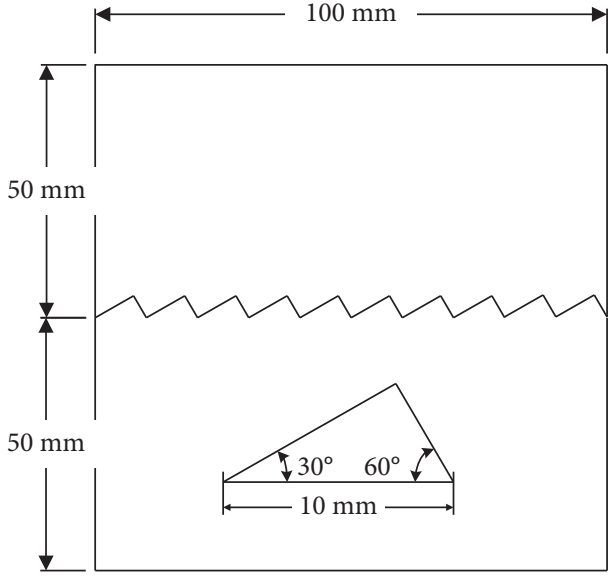

(b)

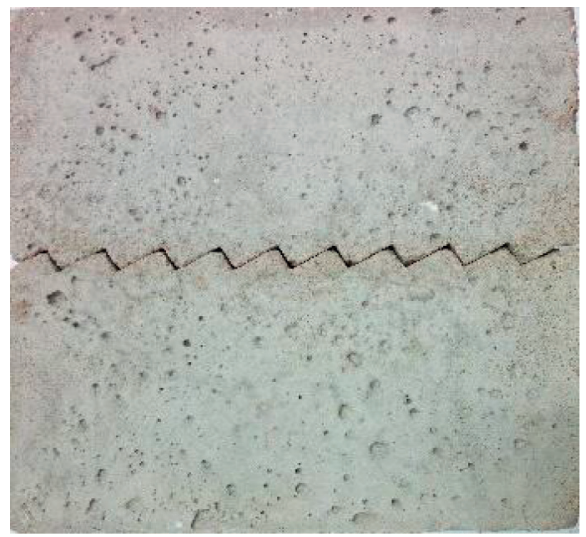

(d)

FIGURE 2: Ichnography of the dentate discontinuities and the sample preparation method: (a) section size of the $10^{\circ}$ structural planes; (b) section size of the $30^{\circ}$ structural planes; (c) sample with a slope angle of $10^{\circ}$; (d) sample with a slope angle of $30^{\circ}$.

samples at different angles under different normal stress is shown in Table 1. Step loading tests and single-stage loading tests were performed on the samples at different stress levels. The shear stress applied in the creep and relaxation tests under step loading were $40 \%, 60 \%, 80 \%, 90 \%$, and $95 \%$ of the average shear strength $\tau_{\max }$ of the structural surface at the respective climbing angle. A schematic of the loading process is shown in Figure 3(a). While conducting the creep and relaxation tests under single-stage loading, a shear stress was selected for the elastic phase and plastic phase of the stressstrain curve because of the creep and relaxation characteristics exhibited by the specimen in both these sections might be different in the past research $[10,13]$. Here, the applied shear stress was $60 \%$ and $90 \%$ of $\tau_{\max }$ at the respective climbing angle, and a schematic of the loading process is shown in Figure 3(b). The details of the tests are as follows: 
TABLE 1: Average shear strength of structural plane.

\begin{tabular}{lcc}
\hline Normal stress $(\mathrm{MPa})$ & Slope angle $\left(^{\circ}\right)$ & Shear strength $\tau_{\text {max }}(\mathrm{MPa})$ \\
\hline 1.962 & 10 & 1.69 \\
1.962 & 30 & 2.60 \\
1.962 & 45 & 3.57 \\
3.924 & 10 & 3.40 \\
3.924 & 30 & 4.40 \\
3.924 & 45 & 5.59 \\
\hline
\end{tabular}

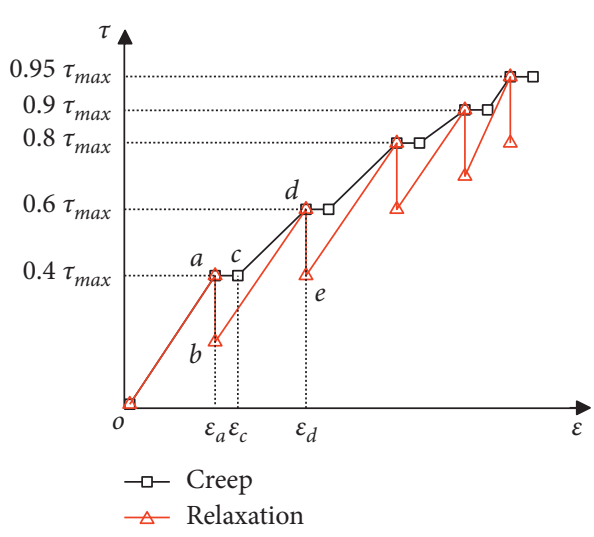

(a)

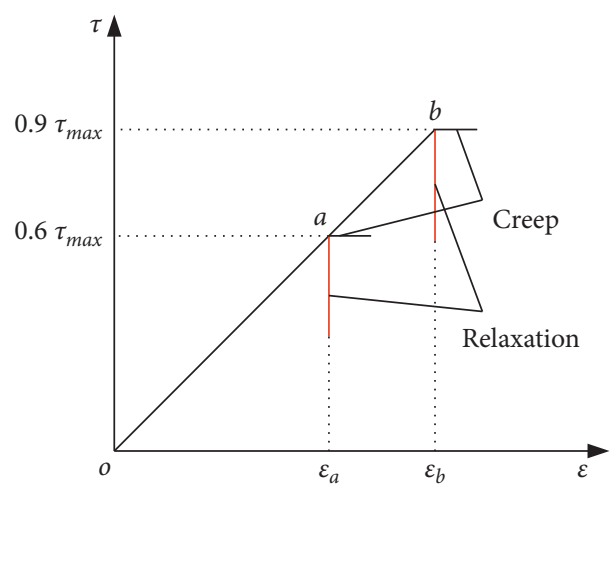

(b)

FIGURE 3: Schematic of the loading process: (a) step loading and (b) single loading.

(1) Figure 3(a) shows the schematic of the creep (relaxation) test under step loading. First, a normal stress of $1.962 \mathrm{MPa}$ or $3.924 \mathrm{MPa}$ is applied and kept constant. After the normal deformation is stabilized, the shear stress is applied at a rate of $0.2 \mathrm{kN} / \mathrm{s}$ to $40 \%$ of the shear strength $\tau_{\max }$, i.e., point a. Subsequently, the shear stress (the strain $\varepsilon_{a}$ generated at this time) is kept stable for $72 \mathrm{~h}$, during which the strain (shear stress) is increased (reduced) from point a to point $c$ (from a point to $b$ point), following which the specified shear stress is applied step by step at the same rate, and the shear stress of each level (strain generated under each level of shear stress) is maintained for $72 \mathrm{~h}$.

(2) Figure 3(b) shows a schematic of the creep test and relaxation test under single loading. First, the normal stress of $1.962 \mathrm{MPa}$ or $3.924 \mathrm{MPa}$ is applied and kept constant. After the normal deformation is stabilized, the shear stress is applied at a rate of $0.2 \mathrm{kN} / \mathrm{s}$ to $60 \%$ (or $90 \%$ ) of the shear strength $\tau_{\max }$, i.e., point a (or point b). The stress (or strain) generated at this time constant is maintained for $72 \mathrm{~h}$.

\section{Processing Method for the Rheological Curve}

The Boltzmann principle is commonly used when test curves of single-stage loading are estimated based on that of step loading, i.e., the total effect due to the external factors at different times in a physical or mechanical system to a certain time in the future is considered. If this effect is linear, it can be processed using the Boltzmann superposition principle. If a certain stress $\sigma=\sigma(t)$ is acting on the object, it can be discretized into a series of single stress pulse acting on the object. According to the Boltzmann superposition principle, the deformation of an object is the comprehensive effect of these pulse stress effects and the general constitutive equation for creep is as follows:

$$
\varepsilon(t)=\frac{\sigma(t)}{E_{0}}+\int_{-\infty}^{t} \sigma(\tau) K(t-\tau) d \tau .
$$

The general integral-type constitutive equation for relaxation can be derived as follows:

$$
\sigma(t)=E_{0} \varepsilon(t)-\int_{-\infty}^{t} \varepsilon(\tau) R(t-\tau) d \tau
$$

where $E_{0}$ is the instantaneous elastic modulus and $K(t-\tau)$ is the creep kernel function, which are determined by the creep properties of the material; $R(t-\tau)$ is the relaxation kernel function, which is determined by the relaxation properties of the material $[15,39]$.

3.1. Finishing Method for the Creep Curve. The step loading is often used in creep tests $[5,10,35,40]$. It is a cascade loading method with a step size of $\Delta \sigma$, which is a kind of step loading method. A schematic of this loading method 
is shown in Figure 4(a). The mapping method is used to establish the superposition principle for the true deformation process by implementing appropriate experimental techniques. As shown in Figure 4(b), it can be applied regardless of whether the after effect is linear or nonlinear. However, this method requires that the deformation into the steady state creep during the lower stage loading is equal to that during the various stages. Yang et al. [39] found that this method is also applicable when the load increments $\Delta \sigma_{i}$ and loading times $\Delta t_{i}$ are not equal at all levels, but the unsteady changes of rheological parameters are not considered.

The creep curve superposition can be expressed as follows:

$$
\begin{aligned}
\varepsilon(\Delta t) & =\frac{n \Delta \sigma}{E_{0}}+n \Delta \sigma \int_{0}^{\Delta t} K(t-\tau) d \tau \\
& =\frac{\sigma}{E_{0}}+\sigma \int_{0}^{\Delta t} K(t-\tau) d \tau .
\end{aligned}
$$

This equation is a creep-type integral equation for stress corresponding to one-time loading $\sigma=n \cdot \Delta \sigma$ in the time interval $\Delta t$, which proves that the effect of step loading is equal to that of the one-time loading $[14,15,39]$.

\subsection{Derivation of the Processing Method for the Relaxation} Curve. Presently, the loading method of most relaxation tests is the same as that of the creep test [18-22], i.e., step loading method used to load the samples in steps of $\Delta \varepsilon$, and the loading method is shown in Figure 5. In this study, the superposition method for the relaxation curve is derived using a method similar to that for arranging the creep data.

The loading process in Figure 5 can be expressed by the following equation:

$$
\begin{aligned}
\varepsilon(t)= & \Delta \varepsilon \theta\left(t-t_{0}\right)+\Delta \varepsilon \theta\left(t-t_{1}\right)+\Delta \varepsilon \theta\left(t-t_{2}\right)+\cdots \\
& +\Delta \varepsilon \theta\left(t-t_{i}\right)+\cdots+\Delta \varepsilon \theta\left(t-t_{n}\right),
\end{aligned}
$$

where $\theta(t)$ is the Heaviside step function.

The $\theta\left(t-t_{i}\right)$ function is defined as follows: if $\left(t-t_{i}\right) \geq 0$, $\theta\left(t-t_{i}\right)=1$; if $\left(t-t_{i}\right) \leq 0, \theta\left(t-t_{i}\right)=0$.

It can be simplified as $\theta\left(t-t_{i}\right)=\theta_{i}$.

Equation (4) is substituted into the relaxation-type integral equation based on the Boltzmann principle of linear superposition, i.e., equation (2) to obtain

$$
\begin{aligned}
\sigma(t)= & \Delta \varepsilon\left(\theta_{0}+\theta_{1}+\theta_{2}+\cdots+\theta_{n}\right) E_{0} \\
& -\int_{0}^{t} \Delta \varepsilon\left(\theta_{0}+\theta_{1}+\theta_{2}+\cdots+\theta_{n}\right) R(t-\tau) d \tau .
\end{aligned}
$$

If the test has been run until $t \geq t_{n}$, then equation (5) is equivalent to

$$
\begin{aligned}
\sigma(t)= & \Delta \varepsilon\left(\theta_{0}+\theta_{1}+\theta_{2}+\cdots+\theta_{n}\right) E_{0}-\int_{t_{0}}^{t} \Delta \varepsilon \theta_{0} R(t-\tau) d \tau \\
& -\int_{t_{1}}^{t} \Delta \varepsilon \theta_{1} R(t-\tau) d \tau-\cdots-\int_{t_{n}}^{t} \Delta \varepsilon \theta_{n} R(t-\tau) d \tau \\
= & n \cdot \Delta \varepsilon E_{0}-\Delta \varepsilon \int_{t_{0}}^{t} \theta_{0} R(t-\tau) d \tau \\
& -\Delta \varepsilon \int_{t_{1}}^{t} \theta_{1} R(t-\tau) d \tau-\cdots-\Delta \varepsilon \int_{t_{n}}^{t} \theta_{n} R(t-\tau) d \tau .
\end{aligned}
$$

It is evident from equation (6) that under the load function of strain shown in equation (4), the total relaxation of material at time $t$ is the sum of the stress increase amount at each level minus the sum of the stress relaxation amounts under the individual strain effects.

The loading time is the same during the test, i.e.,

$$
\left(t_{1}-t_{0}\right)=\left(t_{2}-t_{1}\right)=\left(t_{3}-t_{2}\right)=\cdots=\left(t_{n}-t_{n-1}\right)=\Delta t \text {. }
$$

Define $n \cdot \Delta \varepsilon E_{0}=\varepsilon \cdot E_{0}=\sigma$, and equation (6) can be written as

$$
\sigma(t)=n \cdot \Delta \varepsilon E_{0}-n \Delta \varepsilon \int_{0}^{\Delta t} R(t-\tau) d \tau=\sigma-\varepsilon \int_{0}^{\Delta t} R(t-\tau) d \tau .
$$

This equation is the relaxation-type integral equation for one-time loading $\varepsilon=n \cdot \Delta \varepsilon=n \cdot\left(\Delta \sigma / E_{0}\right)=\sigma / E_{0}$ in the time interval of $\Delta t$, which is similar to the integral equation for creep under one-time loading. The superposition method of the relaxation curve can be obtained by that of creep curve and the derived relaxation-type integral equation of one-time loading. It is evident from Figure 6 that material relaxation can be caused by the loading of the level- 1 strain $\Delta \varepsilon$. From time $t=0$ to $t=t_{1}$, material relaxation occurs under constant strain $\varepsilon=\Delta \varepsilon$, i.e., curve 1. If the next stage of strain $\Delta \varepsilon$ is not added when the test reaches time $t_{1}$, the material stress continues along the dotted line of curve 1 because it has entered the regime of steady state relaxation. At time $t=t_{1}$, the material relaxes after the level-2 strain $\Delta \varepsilon$ is loaded, i.e., curve 2. Considering the effect of loading history and the derived integral equation (equation (8)) of one-time loading, the amount of relaxation generated by the level-2 strain $\Delta \varepsilon$ should be the area enclosed by the relaxation curve (curve (2) of the level-2 strain $\Delta \varepsilon$ and the relaxation curve (curve (1) of the level-1 strain $\Delta \varepsilon$, i.e., the hatched area $\mathrm{A}$ (which can be obtained by shifting the initial point of the relaxation curve of the level-2 strain $\Delta \varepsilon$ to the end point of the level-1 strain $\Delta \varepsilon$ ).Then, the relaxation curve of loading $2 \Delta \varepsilon$ can be obtained by superimposing the hatched area $\mathrm{A}$ on the relaxation curve 1 of the level- 1 strain (the superposition of the hatched area A and the area B), i.e., the curve 3. Based on the Boltzmann superposition principle, the superposition method for the relaxation curve derived according to the creep superposition method can reflect the loading process represented by equation (4). 


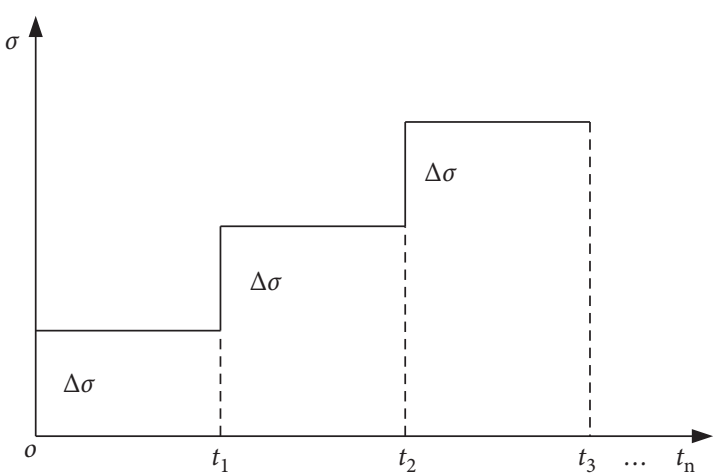

(a)

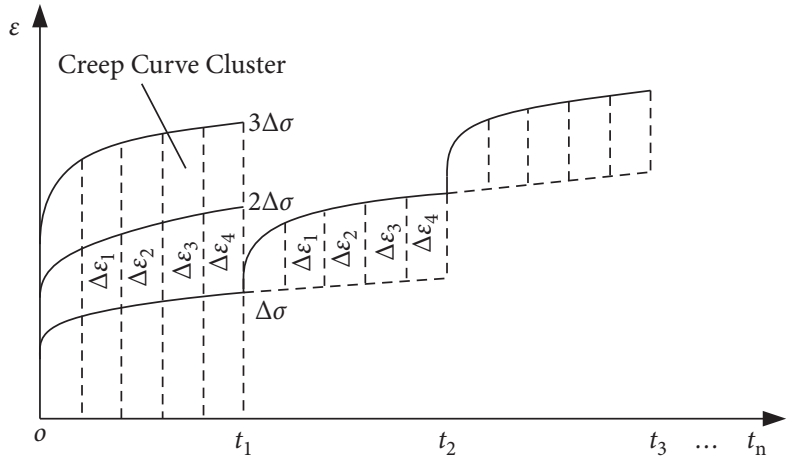

(b)

FIgURE 4: Processing methods for creep curves: (a) the diagram of loading process; (b) deformation diagram.

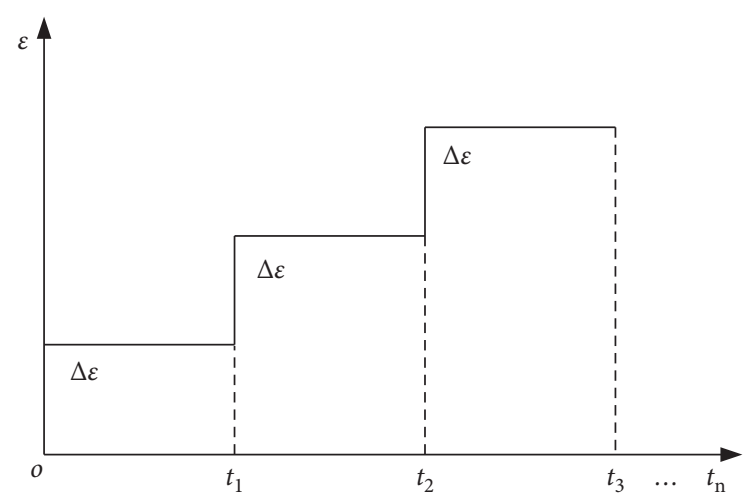

FIGURE 5: Loading process of relaxation test.

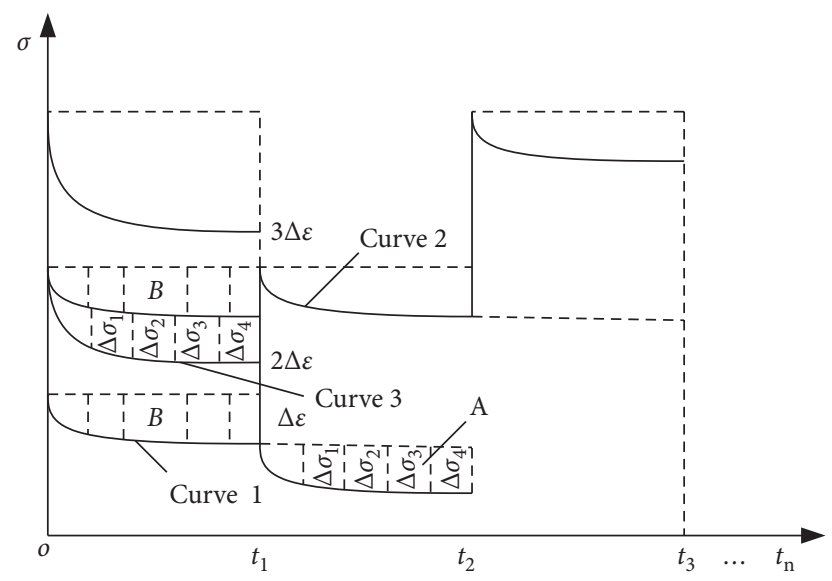

FIgURE 6: Processing methods of relaxation curves.

\section{Analysis of Test Results}

The stacking method for the step loading creep test has been widely studied $[4,6,8,10,13,35,40]$, so it will not be repeated here. The step loading and single-stage loading relaxation experiment are carried out on the $10^{\circ}, 30^{\circ}$, and $45^{\circ}$ structural surfaces to verify the superposition method for relaxation curve derived in the previous section.
4.1. Basic Law of Relaxation. When the normal stress is 3.924 MPa, the entire process curve of step loading relaxation at each climbing angle on the structural surface is shown in Figure 7. When the initial shear stress is within the elastic range and plastic range of the stress-strain curve, respectively, the single-stage loading relaxation curve of $45^{\circ}$ structural plane is shown in Figure 8. The following conclusions can be obtained from Figures 7 and 8:

(1) Figure 7 shows that when step loading relaxation occurs, the initial shear stress level is the same, i.e., the value of $\tau_{0} / \tau_{\max }$ remains the same. Therefore, under the same normal stress, the initial shear stress increases with the increase in the climbing angle, resulting in a stepwise change in the relaxation process curve.

(2) The shape of the relaxation curve under step loading is similar to that under single-stage loading, and the stress significantly decreases with time, which is consistent with the general relaxation law of materials.

(3) During the $72 \mathrm{~h}$ relaxation time, the stress of the step loading and single-stage loading relaxation curve did not relax to zero but approached a stable value. Therefore, the relaxation curve appears as a continuous and incomplete stress relaxation.

(4) The stress relaxation curve can be roughly divided into two phases based on the speed of relaxation: attenuation relaxation and steady-state relaxation.

4.2. Comparison of the Test Results for Single-Stage Loading and Step Loading. Considering the step loading relaxation curve of $10^{\circ}$ structural surface in Figure 7 as an example, the superposition is performed on it according to the superposition method derived in Section 2, i.e., equation (8). Thus, the relaxation curves before and after the superposition can be obtained. The relaxation curve clusters of $10^{\circ}$ structural surface under different shear stress level are shown in Figure 9. Meanwhile, for verifying the superposition results of the relaxation curve and considering that the relaxation 


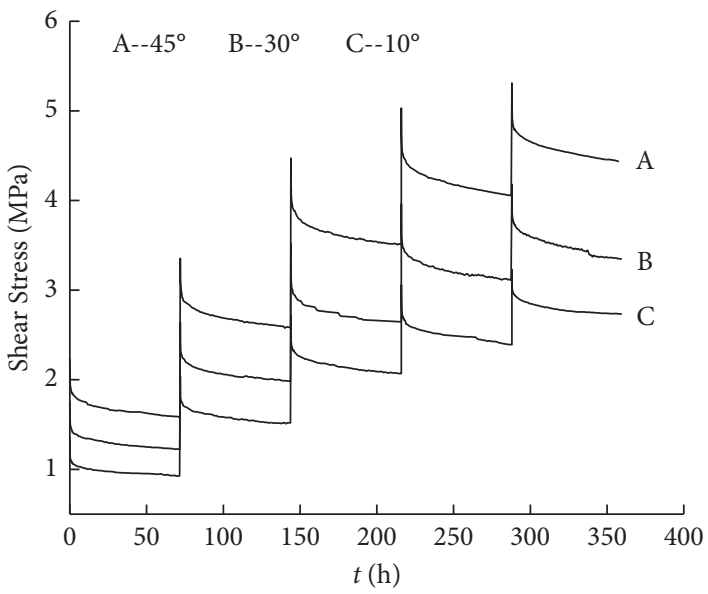

Figure 7: Relaxation curve of the entire process under step loading for $\sigma=3.924 \mathrm{MPa}$.

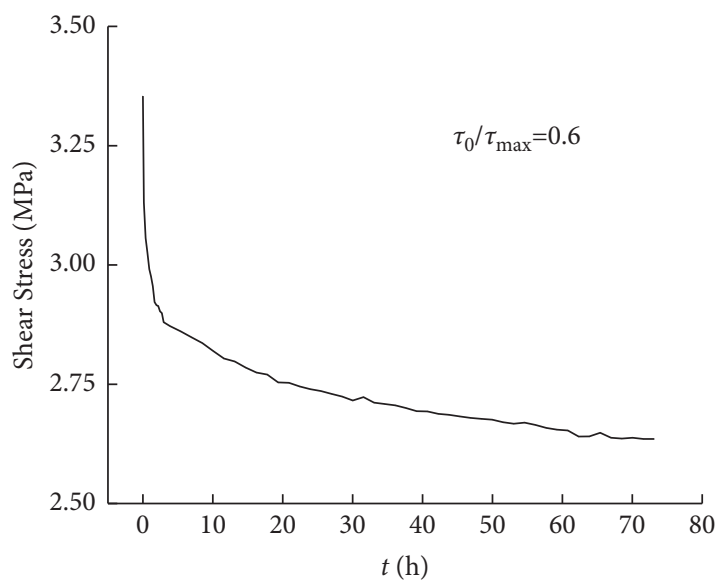

(a)

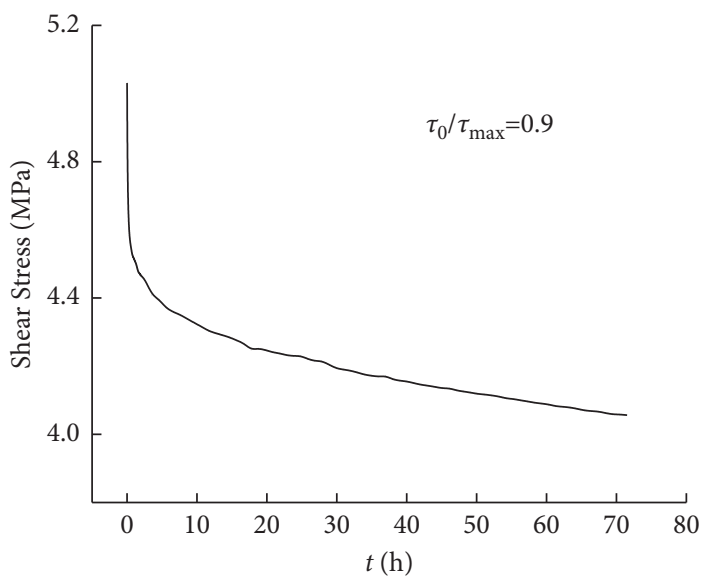

(b)

FIgURE 8: Relaxation curves of $45^{\circ}$ discontinuities under single-stage loading: (a) $\tau_{0} / \tau_{\max }=0.6$; (b) $\tau_{0} / \tau_{\max }=0.9$.

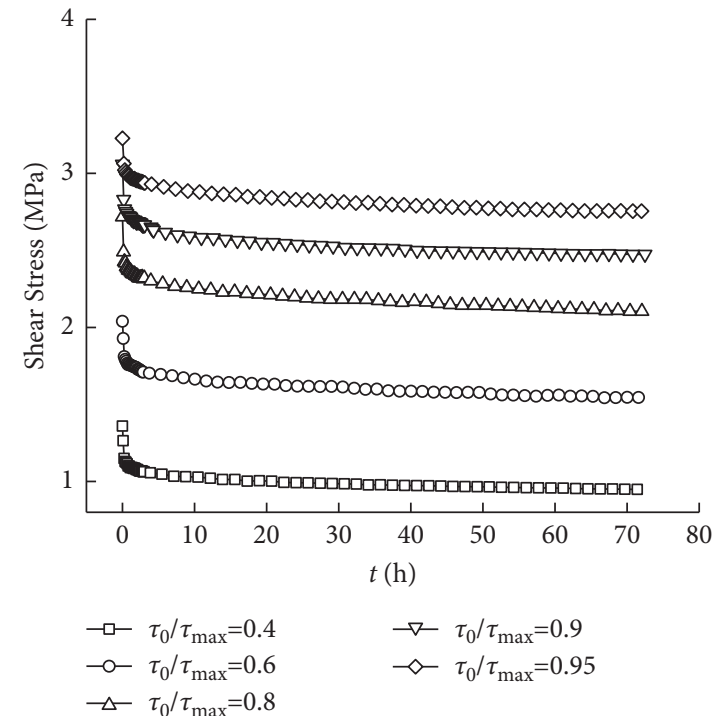

(a)

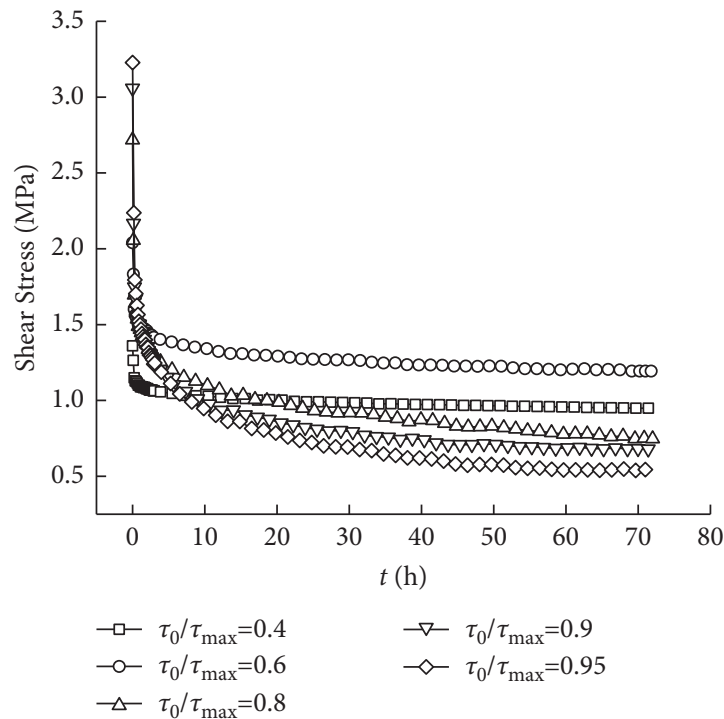

(b)

FIgURe 9: Relaxation curves of $10^{\circ}$ discontinuities under different stress levels: (a) before superposition, $\tau_{0}=3.924 \mathrm{MPa}$; (b) after superposition, $\tau_{0}=3.924 \mathrm{MPa}$. 
characteristics of the specimen in the elastic section and the plastic section might be different, the relaxation curve in elastic deformation sections and plastic deformation section of the $10^{\circ}$ structural planes was taken as example. When the shear stress level of $\tau_{0} / \tau_{\max }$ is 0.6 and 0.9 , the relaxation curves with and without superposition treatment are shown in Figure 10. The relaxation curve loaded to the shear stress level of $\tau_{0} / \tau_{\max }=0.6$ and 0.9 in a single stage is also shown in Figure 10.

At each shear stress level, the difference between initial shear stress $\tau_{0}$ at the beginning of the relaxation test (i.e., at $t=0)$ and the residual stress $\Delta \tau(t)=\tau_{0}-\tau(t)$ after time $t$ is defined as relaxation stress $\Delta \tau(t)=\tau_{0}-\tau(t)$, i.e. $\Delta \tau(t)=\tau_{0}-\tau(t)$, where $t$ is the relaxation time. The following inferences can be drawn from Figures 9 and 10:

(1) Relaxation stress $\Delta \tau(72)$ after $72 \mathrm{~h}$ relaxation at each stress level can be calculated according to Figure 9(a). It is evident that the relaxation curve without superposition exhibits the maximum $\Delta \tau(72)$ at the stress level of $\tau_{0} / \tau_{\max }=0.9$; the relaxation curve after superposition exhibits the maximum $\Delta \tau(72)$ at the stress level of $\tau_{0} / \tau_{\max }=0.95$. This is attributed to the superposition process of relaxation curve considers the effect of the stress levels of the previous levels on the relaxation. Therefore, after the relaxation curve has undergone superposition treatment, the relaxation stress $\Delta \tau(72)$ reaches its maximum at the highest stress level $\tau_{0} / \tau_{\max }=0.95$, which is different from the result without superposition.

(2) Figure 10 shows that when the stress level is $\tau_{0} / \tau_{\max }=0.6$, the maximum relaxation stress $\Delta \tau(72)$ after relaxation curve superposition is greater than that of the untreated curve because the relaxation stress generated under the previous stress level is considered. Besides, it is also greater than the relaxation stress generated by single-stage loading. The results of the relaxation curve with superposition and without superposition are quite different.

(3) The shape of the relaxation curve without superposition is similar to that of the single-stage loading relaxation curve, and the amount of stress relaxation is close. For example, when the stress level is $\tau_{0} / \tau_{\max }=0.6$, the relaxation stress $\Delta \tau(72)$ of the relaxation curve without superposition is about $0.495 \mathrm{MPa}$; the relaxation stress $\Delta \tau(72)$ of the singlestage relaxation curve is about $0.451 \mathrm{MPa}$, and the difference between the relaxation stress of the two curves is $0.044 \mathrm{MPa}$. When the stress level is $\tau_{0} / \tau_{\max }=0.9$, the relaxation stress of the relaxation curve without superposition is about $0.584 \mathrm{MPa}$; the relaxation stress of the single-stage loading relaxation curve is about $0.669 \mathrm{MPa}$, and the difference between the relaxation stress of the two curves is $0.085 \mathrm{MPa}$.

It is evident from the above points that whether it is in the elastic phase (stress level is $\tau_{0} / \tau_{\max }=0.6$ ) or plastic phase (stress level is $\tau_{0} / \tau_{\max }=0.9$ ), the shape of the relaxation curve without superposition is similar to that of the singlestage loading relaxation curve. Moreover, the difference in stress relaxation between the two curves is extremely small, which may be caused by the sample difference.

Although the materials, mixing ratio, and curing conditions used in the sample preparation are same, it is difficult to produce two identical samples. Therefore, it can be considered that the relaxation curve without superposition is equivalent to that with single loading. This implies that when the relaxation test is performed under step loading, the relaxation curve under each level of stress can reflect the relaxation curve when the sample is loaded to the same stress level at a single time without performing superposition on the relaxation curve unlike the step loading creep test.

\section{Difference between Creep Superposition and Relaxation Superposition}

For the creep test performed using the step loading method, the superposition method for creep derived based on the Boltzmann superposition principle has been widely used. Here, the same loading method was used for the creep test to perform the step loading relaxation test, and the superposition method for relaxation was derived based on the Boltzmann superposition principle. However, through experimental comparison, founding that the single-stage loading relaxation curve was very similar to the step loading relaxation curve without superposition, it was quite different from the relaxation curve with superposition treatment. Therefore, if the difference in the test results caused by sample variations is ignored, it can be considered that the step loading relaxation curve without superposition can reflect the relaxation curve of a single-stage load to the same stress level. The cause of this phenomenon was analyzed using the deformation-time curve of creep, the stress-time curve of relaxation, and the stress-strain curve during creep and relaxation processes, which is described as follows. Figures 11 and 12 show the typical creep curve and relaxation curve of step loading, respectively. Considering the $45^{\circ}$ structural surface at normal stress of $1.962 \mathrm{MPa}$ as an example, the shear stress-strain curve during the creep and relaxation tests are shown in Figures 13 and 14, respectively.

It is evident from Figure 11 that the creep deformation during step loading increases with time, and the deformation first increases from point $a$ to point $b$, and then to point $c$, indicating a monotonically increasing trend. The typical relaxation curve shown in Figure 12 indicates that the stress decreases initially and then increases. For example, the first level of strain is applied at $t=0$, and the stress is at point $a$. If the strain is kept constant, it increases with time, and the stress varies from point $a$ to point $b$. At $t=t_{1}$, a second-level strain is applied, and the stress is restored from point $b$ to the level of stress at $t=0$, i.e., point $a^{\prime}$, and then the stress increases to point $c$. Stress does not exhibit a monotonically decreasing or increasing trend. Furthermore, Figures 13 and 14 show that in the creep process, as the stress level increases, the creep deformation increases continuously, exhibiting a monotonically increasing trend. In the relaxation process, 


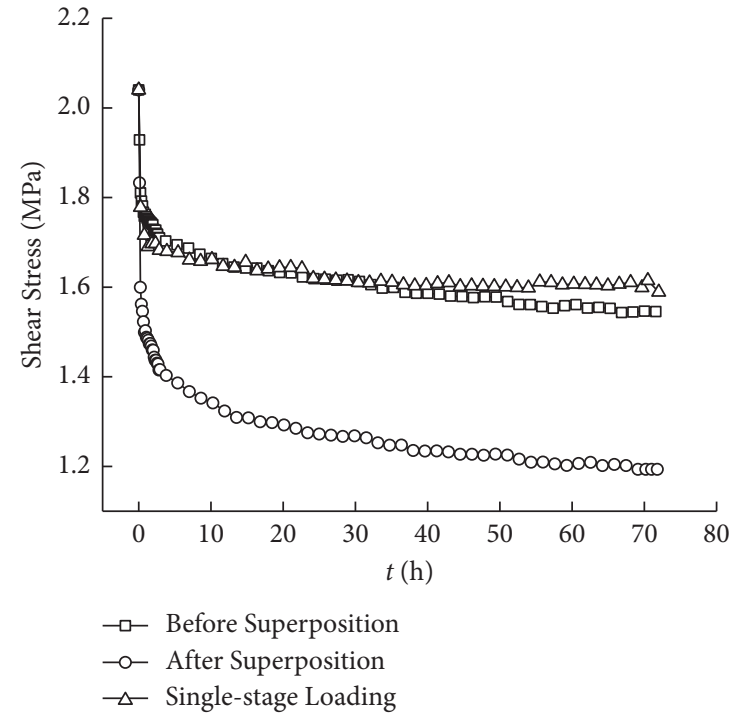

(a)

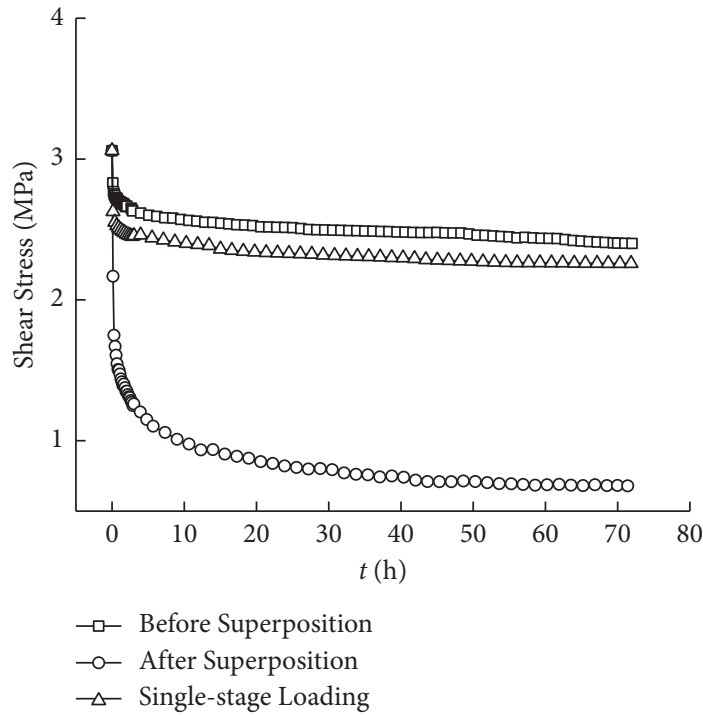

(b)

FiguRE 10: Relaxation curves of $10^{\circ}$ discontinuities by different treatment processes: (a) $\tau_{0} / \tau_{\max }=0.6, \sigma=3.924 \mathrm{MPa}$; (b) $\tau_{0} / \tau_{\max }=0.9$, $\sigma=3.924 \mathrm{MPa}$.

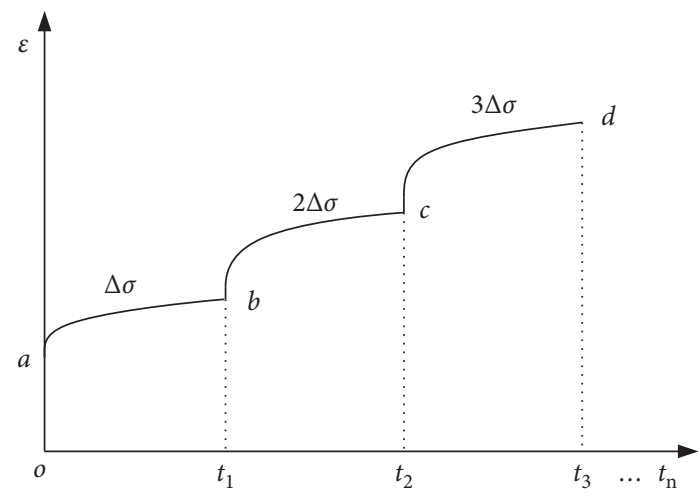

Figure 11: Typical creep curve for step loading.

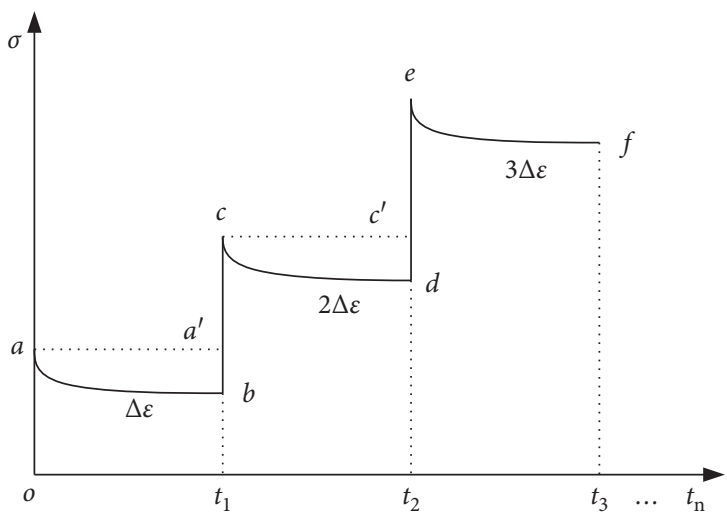

FIGURE 12: Typical relaxation curve for step loading.

the deformation increases monotonically, but the stress first increases, then decreases, and then increases, which is not a monotonic variation.

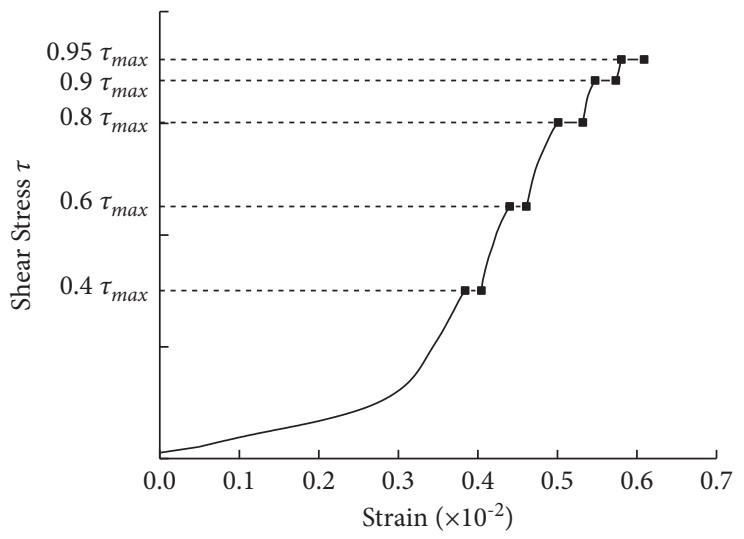

FIGURE 13: Stress-strain curve of $45^{\circ}$ structural plane during creep.

The Boltzmann superposition principle assumes that when the total external effects at different times $\tau$ continue to a certain time $t$ in the future, the principle can be used for mathematical processing only when this effect is linear, i.e., when $\sigma_{1}(t), \sigma_{2}(t)$, and $\sigma_{1}(t)+\sigma_{2}(t)$ are acting on the sample, the strains produced are $\varepsilon_{1}(t), \varepsilon_{2}(t)$, and $\varepsilon_{1}(t)+\varepsilon_{2}(t)$, respectively [15].

The step loading method can be applied regardless of whether the after-effects are linear or nonlinear, but when superposition processing is performed, it is a prerequisite that the external effects on the material are monotonic. For example, in the creep test, the effect of stress on the deformation of the specimen is monotonic; thus, the creep curve can be processed by the superposition method.

In the previous section, based on the relaxation-type integral equation (equation (2)), the superposition method of the relaxation curve was derived according to principle of superposition for the creep method. However, the curve obtained by the relaxation superposition method did not 


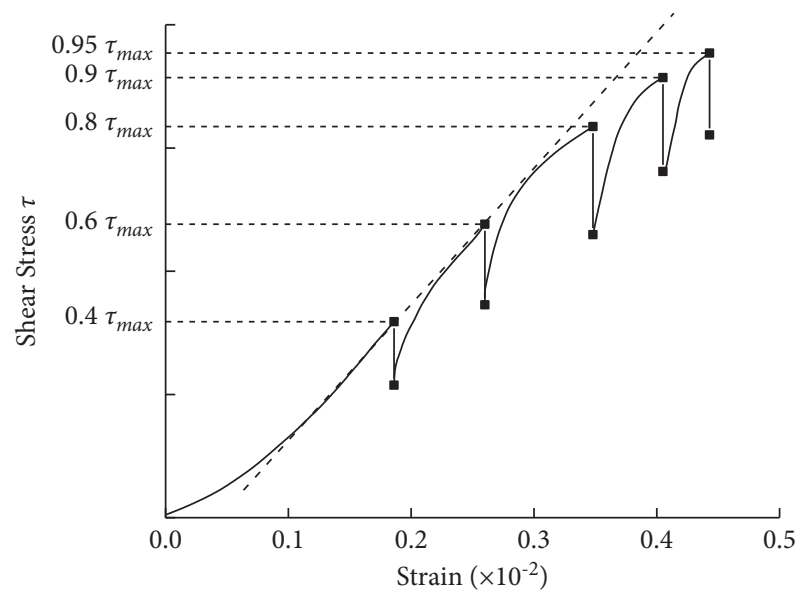

FIgURE 14: Stress-strain curve of the $45^{\circ}$ structural surface during the relaxation process.

match with that of the single-stage loading test for the following reasons:

(1) When the superposition method for the relaxation curve is derived based on the Boltzmann principle and the superposition principle of creep method, the relaxation process needs to satisfy the following conditions: when $\varepsilon_{1}(t), \varepsilon_{2}(t)$, and $\varepsilon_{1}(t)+\varepsilon_{2}(t)$ are acting on the sample, the generated relaxation stress is $\Delta \sigma_{1}(t), \Delta \sigma_{2}(t)$, and $\Delta \sigma_{1}(t)+\Delta \sigma_{2}(t)$, respectively. It can be seen from Figure 9 that the results of relaxation test do not satisfy this condition, so the superposition method for relaxation derived according to the creep superposition method is not applicable here.

(2) In the relaxation process, the effect of strain on stress is not monotonic, but the stress first increases, then decreases, and then again increases, which is different from the monotonically increasing trend observed during the creep process.

\section{Conclusions}

(1) The mathematical basis for the superposition of the relaxation curve under step loading is derived according to the Boltzmann principle. After processing the step loading relaxation curve according to the derived superposition method, it is found that the relaxation curve is significantly different from the single-stage loading relaxation curve at the same shear stress level.

(2) In the step loading test, the relaxation curve of step loading test without superposition at each stress level is basically the same as the single loading stage relaxation curve at the corresponding stress level. It can be considered that when the step loading relaxation test is implemented, the relaxation curve at each level of stress can reflect the relaxation curve when the sample is loaded to the same stress level at a single time without performing superposition on the relaxation curve like the step loading creep test.
(3) The typical creep curve shows that the creep deformation increases monotonically with time, and the curve can be processed by the superposition method, while the typical relaxation curve shows that the stress exhibits a nonmonotonic increasing trend with time during relaxation, which is inconsistent with the Boltzmann principle and cannot been processed by the superposition method.

\section{Data Availability}

The raw/processed data required to reproduce these findings cannot be shared at this time as the data also form part of an ongoing study.

\section{Conflicts of Interest}

The authors declare that they have no conflicts of interest.

\section{Acknowledgments}

This work was supported by the National Natural Science Found Program of China (grant no. 41602287) and the Natural Science Foundation of Henan Province of China (grant no. 182300410161).

\section{References}

[1] M. R. Shen and J. F. Chen, Rock Mechanics, pp. 3-4, Tongji university press, Shanghai, China, 2006.

[2] Q. Y. Zhang, Rheological Test, Rheological Theory and Engineering Application of High Slope Rock Mass, pp. 2-3, Science Press, Beijing, China, 2014.

[3] H. Liu, H. Xie, J. He, M. Xiao, and J. Liu, "Experimental investigation on anisotropic creep characteristics of quartz mica schist," Journal of Southwest Jiaotong University, vol. 50, no. 4, pp. 656-661+697, 2015.

[4] Y. M. Song, T. B. Zhao, and Y. D. Jiang, "Experimental study on the evolution of creep deformation field of rock based on DSCM," Journal of China University of Mining \& Technology, vol. 42, no. 3, pp. 466-470, 2013.

[5] W. J. Xiao, X. J. Wang, C. Chen, Y. L. Zhuo, and Y. X. Li, "Creep strain characteristics of deep limestone under step loading," Journal of Yangtze River Scientific Research Institute, vol. 34, no. 08, pp. 135-138+143, 2017.

[6] W. J. Xie, C. Gong, Y. F. Liu, K. Zhao, and H. G. Li, "Experimental study on creep characteristics of red sandstone under step loading," Journal of Safety Science and Technology, vol. 13, no. 06, pp. 34-39, 2017.

[7] Q. Zhang, Z. Song, J. Wang, Y. Zhang, and T. Wang, "Creep properties and constitutive model of salt rock," Advances in Civil Engineering, vol. 2021, no. 4, pp. 1-29, 2021.

[8] W. Y. Xu, S. Q. Yang, S. L. Yang, S. Y. Xie, J. F. Shao, and Y. F. Wang, "Investigation on triaxial rheological mechanical properties of greenschist specimen(I): experimental results," Rock and Soil Mechanics, vol. 26, no. 4, pp. 531-537, 2005.

[9] M. R. Shen and Q. Z. Zhang, "Study of shear creep characteristics of Greenschist discontinuities," Chinese Journal of Rock Mechanics and Engineering, vol. 29, no. 6, pp. 1149-1155, 2010.

[10] Q. Z. Zhang, M. R. Shen, and W. Q. Ding, "Study on the shear creep characteristics and constitutive model of rock mass 
discontinuity," China Civil Engineering Journal, vol. 44, no. 7, pp. 127-132, 2011.

[11] Z. Wang, L. L. Gu, Q. Z. Zhang, Y. Songlin, and Z. Guokai, "Creep characteristics and prediction of creep failure of rock discontinuities under shearing conditions," International journal of earth science, vol. 109, pp. 945-958, 2020.

[12] H. Lin, X. Zhang, Y. Wang, R. Yong, and X. Fan, "Improved nonlinear nishihara shear creep model with variable parameters for rock-like materials," Advances in Civil Engineering, vol. 2020, no. 3, pp. 1-15, 2020.

[13] C. C. Xia and S. Y. Zhong, "Experimental data processing method in consideration of influence of loading history on rock specimen deformation," Journal of Central-South Institute of Mining and Metallurgy, vol. 20, no. 1, pp. 31-37, 1989.

[14] Z. J. Chen and W. F. Kang, "On the locked in stress, creep and dilatation of rocks, and the constitutive equations," Chinese Journal of Rock Mechanics and Engineering, vol. 10, no. 4, pp. 299-312, 1991.

[15] X. Liu, Introduction to Rock Rheology, pp. 139-143, Geology Publishing House, Beijing, China, 1994.

[16] H. P. Xie and Z. H. Chen, Rock Mechanics, pp. 64-65, Science Press, Beijing, China, 2004.

[17] B. Ladanyi and G. H. Johnston, "Behavior of circular footings and plate Anchors embedded in permafrost," Canadian Geotechnical Journal, vol. 11, no. 4, pp. 531-553, 1974.

[18] H. Yu, M. Zhou, H. Liu, Z. Huang, and T. Jiang, "Experimental investigation on stress relaxation properties of silty mudstone under triaxial compression," Chinese Journal of Rock Mechanics and Engineering, vol. 30, no. 4, pp. 803-811, 2011.

[19] F. M. Chen, Q. G. Hu, and G. Z. Ning, "Silt mudstone stress relaxation characteristics under tri-axial stress condition," Journal of Shandong University, vol. 47, no. 3, pp. 125-129, 2017.

[20] C. Paraskevopoulou, M. Perras, M. Diederichs et al., "The three stages of stress relaxation - observations for the timedependent behaviour of brittle rocks based on laboratory testing," Engineering Geology, vol. 216, pp. 56-75, 2017.

[21] W. F. Zhou and M. R. Shen, "Experimental study of stress relaxation characteristics of regular rock mass discontinuities," Soil Engineering and Foundation, vol. 28, no. 2, pp. 138-141, 2014.

[22] G. H. Tian, M. R. Shen, W. F. Zhou, Y. L. Li, and Z. K. Liu, "Shear relaxation characteristic of serrate structure surface under stepwise loading," Journal of Harbin Institute of Technology, vol. 48, no. 12, pp. 108-113, 2016.

[23] Z. Wang, L. L. Gu, M. R. Sheng, F. Zhangd, G. Zhanga, and $\mathrm{X}$. Wange, "Shear stress relaxation behavior of rock discontinuities with different joint roughness coefficient and stress histories," Journal of Structural Geology, vol. 126, pp. 272-285, 2019.

[24] Q. Zhang, C. Wu, X. Fei, A. Bo, and D. Liu, "Time-dependent behavior of rock joints considering asperity degradation," Journal of Structural Geology, vol. 121, no. 2019, pp. 1-9, 2019.

[25] B. Ladanyi and G. Archambault, "Simulation of shear behavior of a jointed rock mass," Proceedings of the 11th US Symposium on Rock Mechanics, USRMS, American Rock Mechanics Association, New York, NY, USA, 1969.

[26] N. Barton and V. Choubey, "The shear strength of rock joints in theory and practice," Rock Mechanics Felsmechanik Me, vol. 10, no. 1-2, pp. 1-54, 1977.

[27] P. H. S. W. Kulatilake, G. Shou, T. H. Huang, and R. M. Morgan, "New peak shear strength criteria for anisotropic rock joints," International Journal of Rock Mechanics and Mining Science \& Geomechanics Abstracts, vol. 32, no. 7, pp. 673-697, 1995.

[28] C. C. Xia, Z. C. Tang, W. M. Xiao, and Y. L. Song, "New peak shear strength criterion of rock joints based on quantified surface description," Rock Mechanics and Rock Engineering, vol. 47, no. 2, pp. 387-400, 2014.

[29] L.-1. Gu, Z. Wang, F. Zhang, F. Gao, and X. Wang, "Dynamic evolution of shear rate-dependent behavior of rock discontinuity under shearing condition," Journal of Central South University, vol. 28, no. 6, pp. 1875-1887, 2021.

[30] Z. Wang, L. L. Gu, Q. Z. Zhang, and B. A. Jang, "Inluence of initial stress and deformation states on the shear creep behavior of rock discontinuities with diferent joint roughness coeicients," Rock Mechanics and Rock Engineering, 2021.

[31] F. D. Patton, Multiple Modes of Shear Failure in Rock, Proceeding of the1st Congress of International Society of Rock Mechanics, Lisbon PORTUGAL, 1966.

[32] N. Barton, "Review of a new shear-strength criterion for rock joints," Engineering Geology, vol. 7, no. 4, pp. 287-332, 1973.

[33] J. Seidel and C. Haberfield, "The application of energy principles to the determination of the sliding resistance of rock joints," Rock Mechanics and Rock Engineering, vol. 28, no. 4, pp. 211-226, 1995.

[34] H. B. Li, H. Feng, and B. Liu, "Study on strength behaviors of rock joints under different shear deformation velocities," Chinese Journal of Rock Mechanics and Engineering, vol. 25, pp. 2435-2440, 2006.

[35] M. R. Shen and Q. Z. Zhang, "Experimental study of shear deformation characteristics of rock mass discontinuities with regular surface roughness," Chinese Journal of Rock Mechanics and Engineering, vol. 29, no. 4, pp. 713-719, 2010.

[36] D. Huang, R. Q. Huang, and P. Lei, "Shear deformation and strength of through-going saw-tooth rock discontinuity," Journal of China Coal Society, vol. 39, no. 7, pp. 1229-1237, 2014.

[37] H. Zhou, F. Z. Meng, C. Q. Zhang, J. J. Lu, and R. C. Xu, "Experimental study of acoustic emission characteristic of discontinuity under shearing condition," Chinese Journal of Rock Mechanics and Engineering, vol. 34, pp. 2827-2836, 2015.

[38] M. A. Brideau, M. Yan, and D. Stead, "The role of tectonic damage and brittle rock fracture in the development of large rock slope failures," Geomorphology, vol. 103, no. 1, pp. 0-49, 2009.

[39] W. D. Yang, Q. Y. Zhang, F. Chen et al., "Research on nonlinear rheological model of diabase and treatment for creep loading history," Chinese Journal of Rock Mechanics and Engineering, vol. 30, no. 7, pp. 1405-1413, 2011.

[40] A. Liu, M. R. Shen, and J. C. Jiang, "Investigation of the shear stress relaxation characteristics of a structural plane using the isostress cyclic loading method," Geotechnical Testing Journal, vol. 38, no. 2, pp. 219-228, 2015. 